Working Paper No. 558, 2001

Horizontal Mergers Without Synergies May Increase Consumer Welfare

by Johan Stennek

IUI, The Research Institute of Industrial Economics

P.O. Box 5501

SE-114 85 Stockholm

Sweden 


\title{
Horizontal Mergers Without Synergies May Increase Consumer Welfare
}

\author{
Johan Stennek* \\ IUI and CEPR
}

\author{
June 4, 2001
}

\begin{abstract}
Markets with imperfect competition do not induce a cost-minimizing allocation of production between firms. The market's ability to rationalize production is even more limited if costs are private information to firms. Mergers in such markets generate an efficiency gain associated with the pooling of information. Not only may costs be reduced, the price level and price variability may also decline and consumers may thus gain.
\end{abstract}

Keywords: horizontal merger, welfare, asymmetric information

JEL: L10, G34, D43, D82

*I am grateful to Eric van Damme, Henrik Horn, Harald Lang, Lars Persson and Frank Verboven, for helpful discussions. I also thank seminar participants at the Institute for International Economic Studies (Stockholm), Econometric Society European Meeting (Istambul), European Economic Association Anual Meeting (Istambul), and European Association for Research in Industrial Economics (Vienna). I'm grateful to Christina Lönnblad for correcting the language. I thank Jan Wallander och Tom Hedelius Foundation for financial support. Postal address: Research Institute for Industrial Economics (IUI), P.O. Box 5501, SE-114 85 Stockhom, Sweden. 


\section{Introduction}

This paper studies the welfare effects of horizontal mergers in a Cournot duopoly. In particular, I focus on the effect of a merger on consumer welfare through its impact on price. In general, there is a tension between the anti-competitive effects of a merger and efficiency gains. For example, a monopolist can rationalize production, i.e. re-allocate production from high-cost to low-cost plants. However, Farrell and Shapiro (1990) demonstrate that if a merger generates no synergies, defined as cost savings in addition to rationalization, it always raises price and reduces consumer welfare. Although Farrell and Shapiro consider a model with fixed costs, their result is easily generalized to include cost variability as long as information is complete. Since the price (consumer utility) after a merger is higher (lower) than the price (utility) before a merger for every cost contingency, the expected price (utility) must be higher (lower) also when cost varies over time.

In contrast, the present study shows that if firms' costs are private knowledge, a merger may decrease the expected market price, also without synergies. The difference between Farrell and Shapiro's and my results is due to our different models of the pre-merger situation. It is well-known that firms with low marginal costs produce more than firms with high marginal costs, a phenomenon we may call market-induced rationalization. The key point of the present paper is that the market induces less rationalization under incomplete than under complete information. When a firm has a high cost, it plans a small output. Under complete information, the competitors anticipate this and expand their output which, in turn, reduces residual demand to the high-cost firm. Consequently, a high-cost firm also tends to meet a low residual demand and is induced to reduce output even further. Such market induced rationalization cannot occur if costs are private knowledge and thus, mergers generate an extra efficiency gain in markets with asymmetric information, due to pooling of information. 
In markets with fluctuating costs, the price also fluctuates, and consumer welfare cannot be viewed as a function of the price level only. As a further complication, consumers do not unambiguously like or dislike price fluctuations. On the one hand, consumers buy more when the price is low, and less when it is high. They may consume the same average amount as under stable prices, but at a lower average expenditure (price times quantity). On the other hand, price fluctuations create a fluctuating real income, which is disliked if consumers are risk-averse. The risk effect dominates the substitution effect if consumers are strongly risk averse, the good constitutes a large expenditure share and demand is inelastic. I will show that a merger reduces price fluctuations, since the minimum of two costs (which determines the monopoly price) fluctuates less than the sum of two costs (which determines the duopoly price). As a result, a merger may increase consumer welfare, even without reducing the price level.

Implications for merger control and the so-called efficiency defence are spelled out in the concluding section.

\section{The Model}

Consider an industry with two firms $i=1,2$. The good is homogeneous and sold at the market-clearing price, given by the inverse demand function $p=\alpha-q_{1}-q_{2}$. Each firm owns one plant, with constant returns to scale. The marginal cost in a plant, denoted $c_{i}$, is stochastic and distributed on $\left[c_{L}, c_{H}\right]$ with moments $E c=\mu<\alpha$ and $\operatorname{var}\{c\}=\sigma^{2}$. To ensure positive equilibrium quantities, it is assumed that $c_{H} \leq 2 \alpha / 3+\mu / 3$. A merger means that the two plants are operated within the same firm. I assume that the costs are identically and independently distributed over plants, independent of ownership. In reality, idiosyncrasies might at least partly stem from different management styles, so that a merger would increase the correlation between the two costs. If that is the case, the results to follow would be weakened. 
Firms know their own costs before choosing quantities. However, they do not know their competitor's cost, only the cost distribution. Hence, the interaction must be analyzed as a game with incomplete information. In a BayesianNash equilibrium, firm $i$ chooses a supply function $q_{i}\left(c_{i}\right)$, so as to maximize the expected profit, $E_{c_{j}}\left[\alpha-q_{i}-q_{j}\left(c_{j}\right)-c_{i}\right] q_{i}$, given the supply function chosen by the competitor, $q_{j}\left(c_{j}\right)$. Firm $i$ 's equilibrium quantity is given by $q_{i}\left(c_{i}\right)=$ $\frac{1}{3}(\alpha-\mu)-\frac{1}{2}\left(c_{i}-\mu\right)$. The duopoly price is

$$
p^{d}(c)=\frac{1}{3}(\alpha+2 \mu)+\frac{1}{2} \sum_{i}\left(c_{i}-\mu\right) .
$$

The calculations are standard and therefore omitted.

A merger gives the monopolist the opportunity to rationalize production at each point in time. Given a total production, $q$, the monopolist allocates production between plants so as to minimize the production costs, $c_{1} q_{1}(c)+c_{2} q_{2}(c)$. With constant returns to scale, the monopolist simply allocates all production to the plant with the lowest cost. Consequently, the monopolist's technology has constant returns to scale and is characterized by the stochastic marginal cost $\underline{c} \equiv \min \left\{c_{1}, c_{2}\right\}$, which is the so-called first-order statistic. The mean is denoted by $\underline{\mu}$ and variance is denoted by $\underline{\sigma}^{2}$ (Appendix A). The merger does not generate any synergies, defined as cost savings in addition to rationalization. The monopoly chooses the supply function $q^{m}(\underline{c})$ so as to maximize the monopoly profit $(\alpha-q-\underline{c}) q$. The monopoly price is given by

$$
p^{m}(\underline{c})=\frac{1}{2}(\alpha+\underline{\mu})+\frac{1}{2}(\underline{c}-\underline{\mu}) .
$$

The calculations are straight-forward and therefore omitted.

\subsection{The Effect of Merger on the Price Level}

To facilitate the comparison between duopoly and monopoly prices, consider also an intermediary case, namely a hypothetical monopolist that does not allow 
information-sharing between the two plants. Such a monopolist would set

$$
\widetilde{p}(c)=(1 / 2)(\alpha+\mu)+(1 / 2)\left(c_{1}-\mu\right)+(1 / 2)\left(c_{2}-\mu\right)
$$

with $E \tilde{p}=(1 / 2)(\alpha+\mu)$.

The expected monopoly price may be written as $E p^{m}=\frac{1}{2}(\alpha+\mu)+\frac{1}{2}(\underline{\mu}-\mu)$, where the first term equals $E \widetilde{p}$, i.e. the hypothetical expected monopoly price that would result if the monopolist operated the two plants separately. The second term is the reduction in price due to rationalization following from information pooling. The expected duopoly price is given by $E p^{d}=\frac{1}{3} \alpha+\frac{2}{3} \mu$. The change in the expected price as a result of the merger may then be decomposed into

$$
E p^{m}-E p^{d}=\frac{1}{6}(\alpha-\mu)+\frac{1}{2}(\underline{\mu}-\mu) .
$$

The first term is positive and captures the market power effect of the merger, the second is negative and captures the effect of rationalization. Depending on the circumstances, either effect can dominate. If the cost variability is small enough $\sigma^{2}<\frac{2}{9}(\alpha-\mu)^{2}$, a merger raises the expected price. ${ }^{1}$ In particular, if there is no uncertainty, then the present model is a special case of Farrell and Shapiro's model, and a merger will always raise the (expected) price.

Consider now an example where uncertainty is large. The cost is either high $c=1$ or low $c=0$. The probability of a high cost is $\pi=3 / 4$. Let $\alpha=5 / 4$. With a two-point distribution, the price is either high (when $\left(c_{1}+c_{2}\right) / 2=1$ ), medium (when $\left(c_{1}+c_{2}\right) / 2=1 / 2$ ), or low (when $\left.\left(c_{1}+c_{2}\right) / 2=0\right)$. The associated probabilities are $\pi^{2}=9 / 16,2 \pi(1-\pi)=6 / 16$, and $(1-\pi)^{2}=1 / 16$. Under duopoly, prices are 56/48, 32/48 and 8/48, resulting in the expected price $E p^{d}=$ $704 / 768$. Under monopoly, prices are 54/48,30/48 and 30/48, resulting in the expected price $E p^{m}=696 / 768$. Hence,

\footnotetext{
${ }^{1}$ To see this, first note that the price is increased if, and only if, $\mu-\underline{\mu}<(\alpha-\mu) / 3$. Second, use the fact that $\mu-\underline{\mu}<\frac{1}{2} \sqrt{2 \sigma^{2}}$ as is shown in Appendix A, Lemma 3 .
} 
Proposition 1 If costs vary sufficiently, a merger without synergies may reduce the expected price.

Proposition 1 shows that Farrell and Shapiro's Proposition 2 does not apply to incomplete information markets. To better understand the relation between Farrell and Shapiro's results and mine, note that the market induces less rationalization under incomplete than under complete information. When a firm has high costs, it plans a small output. Under complete information, competitors anticipate this and expand their output which, in turn, reduces residual demand to the high-cost firm. Consequently, a high-cost firm also tends to meet a low residual demand, and is thus induced to reduce output even further. Such market induced rationalization cannot occur if costs are private knowledge. Thus, in asymmetric information markets, a merger may generate an additional cost reduction gain and hence, a price reduction gain, due to pooling of information.

Another way of understanding the result is to compare the duopoly price, given by equation (1), with the monopoly price, given by equation (2), to find the cost contingencies under which a monopolist would charge a lower price than a duopoly. Since the monopolist perfectly rationalizes production, the monopoly price depends only on the lowest marginal $\operatorname{cost} \underline{c}=\min \left\{c_{1}, c_{2}\right\}$ at every cost contingency. Under duopoly, however, the high-cost plant also produces and consequently, the duopoly price also depends positively on $\bar{c}=\max \left\{c_{1}, c_{2}\right\}$. Consider the experiment of increasing $\bar{c}$ at a given $\underline{c}$. The duopoly price is then increased without affecting the monopoly price. At some point, the duopoly price exceeds the monopoly price and in particular, it can be shown that $p^{m}(c)<p^{d}(c)$, whenever $\bar{c}>\frac{1}{3} \alpha+\frac{2}{3} \mu$.

Horizontal mergers without synergies may also reduce the price if the firms are involved in tacit collusion prior to the merger, as shown by Verboven (1995). The first reason is that already at the outset, the firms have much market power. The second reason is that, without side-payments, the cartel cannot rational- 
ize production as efficiently as the integrated firm, or even the market under competition.

\subsection{The Effect of Merger on Consumer Welfare}

In markets with fluctuating costs, the price also fluctuates, and consumer welfare cannot be viewed as a function of the price level only; price variability is also important. In fact,

Lemma 1 A cartel decreases price variability.

The proof is relegated to Appendix B. Essentially, this is due to the variability of the price under competition being determined by the variability of the sum of the two (independent) costs, while the variability of the cartel price is determined by the variability of one cost only.

As a further complication, consumers do not unambiguously like or dislike price fluctuations. To be more precise, the linear market demand function can be derived from utility maximization if consumer preferences are identical, quasilinear and quadratic in $q$. Hence, indirect utility is given by $V(p, m)=Y\left(\frac{1}{2}(\alpha-p)^{2}+m\right)$, where $m$ denotes consumer income, and $Y$ is a strictly increasing function. The utility function is assumed to satisfy the von Neumann - Morgenstern property, so that consumer welfare can be evaluated by expected utility. ${ }^{2}$ The change in expected utility, due to a change in price distribution, can be approximated by

$$
E V\left(p^{m}, m\right)-E V\left(p^{d}, m\right) \approx-\left[\frac{E p^{m}-E p^{d}}{\widehat{p}}\right]+\frac{\rho_{p}}{2}\left[\frac{\operatorname{var}\left\{p^{m}\right\}-\operatorname{var}\left\{p^{d}\right\}}{\hat{p}^{2}}\right] .
$$

The formula is derived using a second degree Taylor approximation of the indirect utility function around a price $\widehat{p}$ (Newbery and Stiglitz, 1981). The change in expected utility only depends on the (percentage) change in the expected price,

\footnotetext{
${ }^{2}$ The expected consumer surplus is not a good measure of consumer welfare, unless consumers are risk-netral and the income elasticity of demand is zero (Stennek, 1999).
} 
and a (relative) change in price variability. The only parameter of the approximation, $\rho_{p}$, is the coefficient of relative price risk aversion. ${ }^{3}$ Turnovsky, Shalit, and Schmitz (1980) prove an identity that links price risk aversion to more familiar concepts. In the present context, where the income elasticity of demand is zero,

$$
\rho_{p} \equiv \varepsilon-s \cdot \rho_{m}
$$

where $\varepsilon$ is the price elasticity of demand, $s$ is the expenditure share, and $\rho_{m}$ is the coefficient of relative income risk aversion. Consequently, the price risk-aversion can be decomposed into two effects: the "substitution effect" and the "risk effect." If demand slopes downward, the substitution effect makes consumers favorable to price risk, since they buy a large quantity when the price is low and a small quantity when the price is high. Hence, they might buy the same expected amount as under stable prices, but at a lower expected expenditure (price times quantity). The second effect makes (income) risk-averse consumers also dislike price risk, since variations in the price create variations in real income, in proportion to the expenditure share, and that such income variations are evaluated according to income risk preferences. Consequently, the net effect is ambiguous.

Proposition 2 If consumers are sufficiently risk-averse (toward income risk), and if the expenditure share of the commodity is sufficiently large, a merger increases expected consumer welfare by reducing price variability, independent of the effect on the price level.

The proof follows immediately from the fact that any $\rho_{m}$ is compatible with any given demand function, and that $s$ can be freely varied by varying income, $m$, without affecting demand. By employing a more flexible demand schedule, it is also possible to study the effect of the price and income elasticities of demand.

The paper shows that to assess the welfare effect of a merger, it is important to include the effect on price variability. To evaluate this effect, the size of the

\footnotetext{
${ }^{3}$ Consumer risk-preferences toward price risk are represented by the coefficient of relative price-risk aversion, $\rho_{p}=\left(-V_{p p}(p, m) \cdot p\right) / V_{p}(p, m)$.
} 
market as measured by $s$ and consumer risk-preferences as measured by $\rho_{m}$ must be included.

\section{Conclusions}

The economic literature provides several arguments why competition authorities should balance the anti-competitive effects of mergers against their efficiency gains. Williamson (1968) uses a cost-benefit analysis to show that horizontal mergers may increase social welfare if they save on costs. Farrell and Shapiro (1990) study merger in a Cournot model and show that consumers may also gain, if the merger generates cost synergies. The economic literature also provides practical tools for making the trade-off between anti-competitive effects and efficiency gains, such as simulation analysis (Werden and Froeb, 1994; Ivaldi and Verboven, 2001) and econometric techniques to estimate the pass-on of firmspecific cost savings (Ashenfelter, Ashmore, Baker and McKerman, 1998).

The economic analysis in this field has had some impact on policy. Today, several merger control systems in the OECD allow a so-called efficiency defence, an example of which is the US with its Federal Merger Guidelines. The first releases of the Guidelines in 1968 and 1982 opposed efficiency considerations unless under exceptional circumstances. The later releases of the Guidelines in 1984 and 1992 (which is still in use today) reveal a more sympathetic treatment of efficiency claims. In Europe, however, merger policy is still unclear as concerns the role of efficiencies (Röller, Stennek and Verboven, 2000; Stennek and Verboven, 2001).

The present paper demonstrates the importance of a set of previously neglected circumstances when assessing the effects of horizontal mergers. In markets where firms' costs are private information, mergers generate efficiency gains associated with pooling of information. To take these gains into account, it is necessary to assess the degree to which cost shocks are idiosyncratic and information is asymmetric; the degree of consumer risk-aversion and the size of the 
market. Since information is generally imperfect, and may often be very limited, these extensions seem to be of importance for a complete assessment of mergers.

\section{A Preliminaries}

Let $c_{1}$ and $c_{2}$ be identically and independently distributed according to the cumulative distribution function $F$ and density $f$. Denote the mean by $\mu$ and the variance by $\sigma^{2}$. The first-order statistic $\underline{c} \equiv \min \left\{c_{1}, c_{2}\right\}$ has density $\underline{f}(\underline{c})=$ $2[1-F(\underline{c})] f(\underline{c})$. The mean is denoted by $\underline{\mu}$ and the variance by $\underline{\sigma}^{2}$. The second-order statistic $\bar{c} \equiv \max \left\{c_{1}, c_{2}\right\}$ has density $\bar{f}(\bar{c})=2 F(\bar{c}) f(\bar{c})$. The mean is denoted by $\bar{\mu}$ and the variance by $\bar{\sigma}^{2}$.

\section{Lemma 2}

(i) $\frac{1}{2} \bar{\mu}+\frac{1}{2} \underline{\mu}=\mu$;

(ii) $(\mu-\bar{\mu})^{2}=(\mu-\underline{\mu})^{2}$;

(iii) $\sigma^{2}=\frac{1}{2} \bar{\sigma}^{2}+\frac{1}{2} \underline{\sigma}^{2}+\frac{1}{2}(\mu-\bar{\mu})^{2}+\frac{1}{2}(\mu-\underline{\mu})^{2}$.

Proof. $\quad$ To see (i): $\frac{1}{2} \bar{\mu}+\frac{1}{2} \underline{\mu}=\frac{1}{2} E \bar{c}+\frac{1}{2} E \underline{c}=\frac{1}{2} E c_{1}+\frac{1}{2} E c_{2}$. Hence $\frac{1}{2} \bar{\mu}+$ $\frac{1}{2} \underline{\mu}=\frac{1}{2} \mu+\frac{1}{2} \mu=\mu$. To see (ii): use $\bar{\mu}+\underline{\mu}=2 \mu$. Hence $\bar{\mu}-\mu=\mu-\underline{\mu}$. To see (iii): $\sigma^{2}=\frac{1}{2} \sigma^{2}+\frac{1}{2} \sigma^{2}=\frac{1}{2} E\left(c_{1}-\mu\right)^{2}+\frac{1}{2} E\left(c_{2}-\mu\right)^{2}=\frac{1}{2} E(\bar{c}-\mu)^{2}+$ $\frac{1}{2} E(\underline{c}-\mu)^{2}$ Hence $\sigma^{2}=\frac{1}{2} E((\bar{c}-\bar{\mu})+(\bar{\mu}-\mu))^{2}+\frac{1}{2} E((\underline{c}-\underline{\mu})+(\underline{\mu}-\mu))^{2}$, and $\sigma^{2}=\frac{1}{2} E\left((\bar{c}-\bar{\mu})^{2}+2(\bar{c}-\bar{\mu})(\bar{\mu}-\mu)+(\bar{\mu}-\mu)^{2}\right)+$ $+\frac{1}{2} E\left((\underline{c}-\underline{\mu})^{2}+2(\underline{c}-\underline{\mu})(\underline{\mu}-\mu)+(\underline{\mu}-\mu)^{2}\right)$. Hence, $\sigma^{2}=\frac{1}{2} \bar{\sigma}^{2}+\frac{1}{2} \underline{\sigma}^{2}+\frac{1}{2}(\mu-\bar{\mu})^{2}+$ $\frac{1}{2}(\mu-\underline{\mu})^{2}$.

\section{Lemma 3}

(i) $\bar{\mu}-\underline{\mu}<\sqrt{2 \sigma^{2}}$;

(ii) $\mu-\underline{\mu}<\frac{1}{2} \sqrt{2 \sigma^{2}}$.

Proof. To see (i): $\bar{\mu}-\underline{\mu}=E \bar{c}-E \underline{c}=E\left|c_{1}-c_{2}\right|$. Note also that $\left|c_{1}-c_{2}\right|=$ $\sqrt{\left(c_{1}-c_{2}\right)^{2}}$ and hence, $\bar{\mu}-\underline{\mu}=E \sqrt{\left(c_{1}-c_{2}\right)^{2}}$. Since $\sqrt{\cdot}$ is strictly concave, 
then by Jensen's inequality, we have $E \sqrt{\left(c_{1}-c_{2}\right)^{2}}<\sqrt{E\left(c_{1}-c_{2}\right)^{2}}$, and hence $\bar{\mu}-\underline{\mu}<\sqrt{E\left(c_{1}-c_{2}\right)^{2}}$. Further $E\left(c_{1}-c_{2}\right)^{2}=E\left(\left(c_{1}-\mu\right)-\left(c_{2}-\mu\right)\right)^{2}=2 \sigma^{2}$ and hence, $\bar{\mu}-\underline{\mu}<\sqrt{2 \sigma^{2}}$. To see (ii): Combine Lemma 2 (i) and Lemma 3 (i) to get $(2 \mu-\underline{\mu})-\underline{\mu}<\sqrt{2 \sigma^{2}}$, and hence $\mu-\underline{\mu}<\frac{1}{2} \sqrt{2 \sigma^{2}}$.

\section{B Proof of Lemma 1}

Since prices are linear in costs, monopoly price variability is

$$
\operatorname{var}\left\{p^{m}\right\}=\left(\partial p^{m} / \partial \underline{c}\right)^{2} \operatorname{var}\{\underline{c}\}=\frac{1}{4} \underline{\sigma}^{2},
$$

and duopoly price variability is

$$
\operatorname{var}\left\{p^{d}\right\}=\sum_{i=1}^{2}\left(\partial p^{d} / \partial c_{i}\right)^{2} \operatorname{var}\left\{c_{i}\right\}=2 \frac{1}{4} \sigma^{2}
$$

Hence, three factors determine the size of the variances: the sensitivity of price to cost shocks, the number of operated plants, and the variability of cost. First, the sensitivity of the price to cost shocks is the same in asymmetric information duopoly as in monopoly, that is $\partial p^{d} / \partial c_{i}=\partial p^{m} / \partial \underline{c}=1 / 2$. Second, a merger reduces the number of plants operated at each point in time, which unambiguously tends to decrease price variability. Third, the variability of $\underline{c}$ might be larger as well as smaller than the variability of $c_{i}$, depending on the exact distribution. Consequently, there are two potentially opposing effects.

To facilitate the comparison, the variability of the duopoly price is rewritten as

$$
\begin{aligned}
\operatorname{var}\left\{p^{d}\right\} & =\left(\partial p^{m} / \partial \underline{c}\right)^{2} \operatorname{var}\{\underline{c}\}+\left(\partial p^{m} / \partial \underline{c}\right)\left(\partial p^{m} / \partial \bar{c}\right) \operatorname{cov}\{\underline{c}, \bar{c}\}+\left(\partial p^{m} / \partial \bar{c}\right)^{2} \operatorname{var}\{(\underline{\theta}\}) \\
& =\frac{1}{4} \underline{\sigma}^{2}+\frac{1}{2} \operatorname{cov}\{\underline{c}, \bar{c}\}+\frac{1}{4} \bar{\sigma}^{2} .
\end{aligned}
$$

Since $\operatorname{cov}\{\underline{c}, \bar{c}\}>0$ and $\bar{\sigma}^{2}>0$, it follows that $\operatorname{var}\left\{p^{m}\right\}<\operatorname{var}\left\{p^{d}\right\}$.

\section{References}


Ashenfelter, Orley; Ashmore, David; Baker, Jonathan B.; Mc Kernan, SigneMary: Identifying the Firm-Specific Pass-Through Rate, FTC Discussion paper, 1998.

Farrell, Joseph; Shapiro, Carl: Horizontal Mergers: An Equilibrium Analysis, American Economic Review; 80(1), March 1990, 107-26.

Ivald, Marc; Verboven, Frank: Quantifying the Effects of Horizontal Merger in European Competition Policy, mimeo. University of Leuven, 2001.

Newbery, David M. G.; Stiglitz, Joseph E.: The Theory of Commodity Price Stabilization: A Study in the Economics of Risk, Clarendon Press, Oxford, 1981.

Röller, Lars-Hendrik; Stennek, Johan; Verboven, Frank: Efficiency Gains from Mergers, Report to the European Commission (EC Contract II/98/003), February 2000.

Stennek, Johan: The Expected Consumer's Surplus as a Welfare Measure, Journal of Public Economics, 73(2), August 1999, 265-288.

Stennek, Johan; Verboven, Frank: Merger Control and Enterprise Competitiveness, Report to the European Commission (EC Contract III/99/065), April 2001.

Turnovsky, Stephen J.; Shalit, Haim; Schmitz, Andrew: Consumer's Surplus, Price Instability, and Consumer Welfare, Econometrica; 48(1), Jan. 1980, 135-52.

Verboven, Frank: Corporate Restructuring in a Collusive Oligopoly, International Journal of Industrial Organization; 13(3), September 1995, 335-54. 
Werden, Gregory J.; Froeb, Luke M., The Effects of Mergers in Differentiated Products Industries: Logit Demand and Merger Policy, Journal of Law, Economics, and Organization; 10(2), October 1994, pages 407-26.

Williamson, Oliver E.: Economies as an Antitrust Defence: The Welfare Tradeoffs, American Economic Review; 58, 1968, 18-36.

Document: Fash.tex 